\title{
Liquid Chromatography: Introduction to Method Development
}

\author{
Lane C. Sander \\ National Institute of Standards and Technology, \\ Gaithersburg, MD 20899, USA \\ lane.sander@nist.gov
}

Video DOI: http://doi.org/10.18434/T4DK5T

Key words: liquid chromatography; method development; quantitation; metrology; column selection; gradient elution; isocratic elution.

Accepted: March 6, 2017

Published: March 8, 2017

https://doi.org/10.6028/jres.122.018

\section{Summary}

The development of fit-for-purpose analytical methods is an essential component of chemical metrology. This presentation will provide guidance on approaches for establishing robust analytical methods, particularly with applications to liquid chromatography. Because nearly every measurement need represents unique challenges, this guidance is necessarily general in scope. A stepwise process is outlined for the development and implementation of an analytical procedure. The presentation includes the following topics that are not limited to liquid chromatography, but are generally applicable to most instrumental methods.

1. Objectives of the analysis. Depending on the goals, the details of the method used will vary significantly.

2. Sample Properties. The physical and chemical properties of both the sample (matrix) and the analytes are central to the development of analytical methods.

3. Choice of instrumental method

4. Sample processing

5. Basis for quantitation

Details specific to the development of methods based on liquid chromatography are provided in the second half of the presentation. Recommendations are made concerning initial column selection and the choice of isocratic or gradient elution approaches. Different decisions may be required that depend on the measurement challenge. Alternatives are discussed that can be explored when measurement goals are not initially achieved. ${ }^{1}$

\footnotetext{
${ }^{1}$ Contribution of the National Institute of Standards and Technology. Not subject to copyright. Certain commercial equipment, instruments, or materials are identified to specify adequately the experimental procedure. Such identification does not imply recommendation or endorsement by the National Institute of Standards and Technology, nor does it imply that the materials or equipment identified are the best available for the purpose.
} 